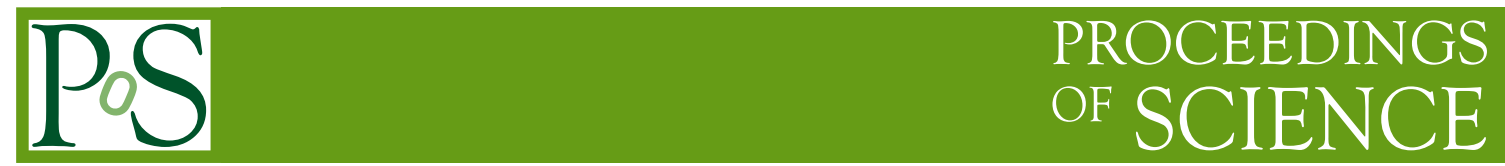

\title{
LFV: Where are we?
}

\section{Paride Paradisi*}

Technical University Munich, Physics Department, D-85748 Garching, Germany

E-mail: paride.paradisi@ph.tum.de

The synergy and interplay of high energy data with flavour precision data are expected to shed some light on the "Flavour Problem". Lepton Flavor Violating processes like $\mu \rightarrow e \gamma$ represent "golden channels" where to look for New Physics effects, given their high New Physics sensitivity and the outstanding experimental progress we expect in the upcoming years. If LFV will be observed, the next crucial step will be to trace back the New Physics model at work by means of a correlated analysis of various observables.

The Xth Nicola Cabibbo International Conference on Heavy Quarks and Leptons,

October 11-15, 2010

Frascati (Rome) Italy

${ }^{*}$ Speaker. 


\section{Introduction}

The most important achievement we expect to reach at the beginning of the LHC era is the understanding of the underlying mechanism accounting for the electroweak symmetry breaking, in particular, whether the Higgs mechanism is realized in nature or not. Moreover, the LHC is also expected to shed light on the hierarchy problem, since a natural solution of it calls for a $\mathrm{TeV}$ scale New Physics (NP).

On the other hand, low-energy flavour physics observables provide the most powerful tool to unveil the symmetry properties of the NP theory that will emerge at the LHC, if any. In fact, highprecision measurements at the LHC are made typically challenging by the huge background and by irreducible hadronic uncertainties.

The last decade has established that flavour-changing and CPV processes in $B_{s, d}$ and $\mathrm{K}$ systems are well described by the SM. The same is true for electroweak precision tests. This implies automatically tight constraints on flavour-changing phenomena beyond the SM and a potential problem for a natural solution of the hierarchy problem.

On general ground, the main lesson we learned so far from the flavour data is that a $\mathrm{TeV}$ scale NP must have a highly non-generic flavour structure in order to satisfy all the existing constraints. Moreover, in order to avoid fine tuning of parameters, natural protection mechanisms suppressing FCNCs generated by NP are required. Famous examples of such mechanisms are MFV, alignment and degeneracy, as arising from abelian and non-abelian flavour symmetries.

The SM mechanism of flavour mixing has been tested with high accuracy in the quark sector, where all flavour-violating phenomena seem to be well described by the SM Yukawa interaction [1]. Flavour mixing has been observed also in the neutrino sector, indicating the existence of a nonvanishing neutrino mass matrix which cannot be accommodated within the SM.

However, the origin of flavour is still far from being established. The most important open questions can be summarized as follow:

- Which is the organizing principle behind the observed pattern of fermion masses and mixing angles?

- Are there extra sources of flavour symmetry breaking beside the SM Yukawa couplings which are relevant at the $\mathrm{TeV}$ scale?

The search for LFV in charged leptons is probably the most interesting goal of flavour physics in the next few years. The observation of neutrino oscillations has clearly demonstrated that lepton flavour is not conserved; however, the smallness of neutrino masses provides a strong indication that neutrinos are generated by an underlying dynamics that violates also the total lepton number. The question is if LFV effects can be visible also in other sectors of the theory, or if we can observe LFV in processes which conserve the total lepton number.

\section{Experimental status for LFV}

The status of searches for some selected LFV channels in $\tau$ and $\mu$ decays is summarized in Table 1. 


\begin{tabular}{|l|l|l|l|}
\hline Process & Present Bounds & Expected Future Bounds & Future Experiments \\
\hline \hline $\operatorname{BR}(\mu \rightarrow e \gamma)$ & $1.2 \times 10^{-11}$ & $\mathscr{O}\left(10^{-13}-10^{-14}\right)$ & MEG, PSI \\
$\operatorname{BR}(\mu \rightarrow e e e)$ & $1.1 \times 10^{-12}$ & $\mathscr{O}\left(10^{-13}-10^{-14}\right)$ & $?$ \\
$\operatorname{BR}(\mu \rightarrow e$ in Nuclei $(\mathrm{Ti}))$ & $1.1 \times 10^{-12}$ & $\mathscr{O}\left(10^{-18}\right)$ & J-PARC \\
$\operatorname{BR}(\tau \rightarrow e \gamma)$ & $1.1 \times 10^{-7}$ & $\mathscr{O}\left(10^{-8}\right)$ & SuperB \\
$\operatorname{BR}(\tau \rightarrow e e e)$ & $2.7 \times 10^{-7}$ & $\mathscr{O}\left(10^{-8}\right)$ & SuperB \\
$\operatorname{BR}(\tau \rightarrow e \mu \mu)$ & $2 . \times 10^{-7}$ & $\mathscr{O}\left(10^{-8}\right)$ & SuperB \\
$\operatorname{BR}(\tau \rightarrow \mu \gamma)$ & $6.8 \times 10^{-8}$ & $\mathscr{O}\left(10^{-8}\right)$ & SuperB \\
$\operatorname{BR}(\tau \rightarrow \mu \mu \mu)$ & $2 \times 10^{-7}$ & $\mathscr{O}\left(10^{-8}\right)$ & LHCb \\
$\operatorname{BR}(\tau \rightarrow \mu e e)$ & $2.4 \times 10^{-7}$ & $\mathscr{O}\left(10^{-8}\right)$ & SuperB \\
\hline
\end{tabular}

Table 1: Present [2] and upcoming experimental limits on various leptonic processes at 90\% C.L.

In particular, the MEG experiment at PSI [3] should be able to test $\operatorname{Br}(\mu \rightarrow e \gamma)$ at the level of $\mathscr{O}\left(10^{-13}\right)$, and the Super Flavour Factory [4] is planned to reach a sensitivity for $\operatorname{Br}(\tau \rightarrow \mu \gamma)$ of $\mathscr{O}\left(10^{-9}\right)$ and also the planned resolution of SuperKEKB for $\tau \rightarrow \mu \gamma$ is of $\mathscr{O}\left(10^{-8}\right)$. An impressive improvement is also expected for the upper bound on $\mu-e$ conversion in Ti. The dedicated J-PARC and PRISM/PRIME experiment [5] should reach the sensitivity of $\mathscr{O}\left(10^{-18}\right)$, almost six orders of magnitude better than the current upper bound from SINDRUM II at PSI [6].

\section{Flavour Violation in Charged Lepton Decays}

In the SM with massive neutrinos, the branching ratio for LFV processes like $\mu \rightarrow e \gamma$ is of order $\operatorname{Br}(\mu \rightarrow e \gamma)_{\mathrm{SM}} \approx 10^{-54}$, to be compared with the $90 \%$ C.L. upper bound from the MEGA Collaboration [7] $\operatorname{Br}(\mu \rightarrow e \gamma)<1.2 \cdot 10^{-11}$. Therefore any observation of LFV would be a clear signal of NP.

On general grounds, if the breaking of the total lepton number occurs at a very high energy scale $\left(\Lambda_{\mathrm{LN}}>10^{12} \mathrm{GeV}\right)$, as expected by the smallness of neutrino masses, and the theory has new degrees of freedom carrying lepton-flavour quantum numbers around the TeV scale $\left(\Lambda_{\mathrm{LFV}}<\right.$ $10^{4} \mathrm{GeV}$ ), then $\mu \rightarrow e \gamma$ should be visible. Indeed, employing an effective theory approach with a minimal breaking of lepton flavour, we find [8]

$$
\mathscr{B}(\mu \rightarrow e \gamma) \approx 10^{-13}\left(\frac{\Lambda_{\mathrm{LN}}}{10^{13} \mathrm{GeV}}\right)^{4}\left(\frac{10^{4} \mathrm{GeV}}{\Lambda_{\mathrm{LFV}}}\right)^{4}
$$

A typical concrete example where this occurs is the MSSM with heavy right-handed neutrinos, where renormalization-group effects generate LFV entries in the left-handed slepton mass matrices at the TeV scale [9]. Once non-vanishing LFV entries in the slepton mass matrices are generated, LFV rare decays are naturally induced by one-loop diagrams with the exchange of gauginos and sleptons. The flavour-conserving component of the same diagrams induces a non-vanishing contribution to the anomalous magnetic moment of the muon, $\Delta a_{\mu}=\left(g_{\mu}-g_{\mu}^{\mathrm{SM}}\right) / 2$. As shown in Fig. 1, 


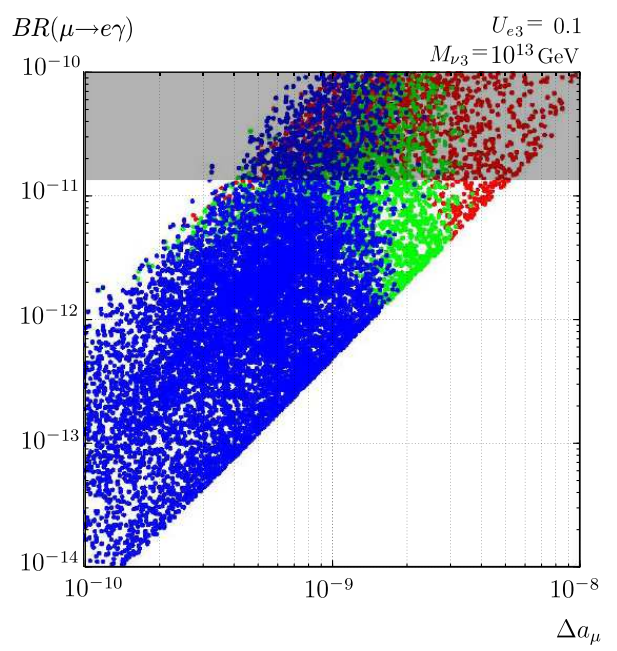

Figure 1: Correlation between $\mathscr{B}(\mu \rightarrow e \gamma)$ and $\Delta a_{\mu}$ in the MSSM with heavy RH neutrinos [10].

\begin{tabular}{|c|c|c|c|}
\hline ratio & LHT & MSSM & SM4 \\
\hline \hline$\frac{B r(\mu \rightarrow e e e)}{B r(\mu \rightarrow e \gamma)}$ & $0.02 \ldots 1$ & $\sim 2 \cdot 10^{-3}$ & $0.06 \ldots 2.2$ \\
\hline$\frac{B r(\tau \rightarrow e e e)}{B r(\tau \rightarrow e \gamma)}$ & $0.04 \ldots 0.4$ & $\sim 1 \cdot 10^{-2}$ & $0.07 \ldots 2.2$ \\
\hline$\frac{B r(\tau \rightarrow \mu \mu \mu)}{B r(\tau \rightarrow \mu \gamma)}$ & $0.04 \ldots 0.4$ & $\sim 2 \cdot 10^{-3}$ & $0.06 \ldots 2.2$ \\
\hline$\frac{B r(\tau \rightarrow e \mu \mu)}{B r(\tau \rightarrow e \gamma)}$ & $0.04 \ldots 0.3$ & $\sim 2 \cdot 10^{-3}$ & $0.03 \ldots 1.3$ \\
\hline$\frac{B r(\tau \rightarrow \mu e e)}{B r(\tau \rightarrow \mu \gamma)}$ & $0.04 \ldots 0.3$ & $\sim 1 \cdot 10^{-2}$ & $0.04 \ldots 1.4$ \\
\hline$\frac{B r(\tau \rightarrow e e e)}{B r(\tau \rightarrow e \mu \mu)}$ & $0.8 \ldots 2$ & $\sim 5$ & $1.5 \ldots 2.3$ \\
\hline$\frac{B r(\tau \rightarrow \mu \mu \mu)}{B r(\tau \rightarrow \mu e e)}$ & $0.7 \ldots 1.6$ & $\sim 0.2$ & $1.4 \ldots 1.7$ \\
\hline$\frac{\mathrm{R}(\mu \mathrm{Ti} \rightarrow e \mathrm{Ti})}{B r(\mu \rightarrow e \gamma)}$ & $10^{-3} \ldots 10^{2}$ & $\sim 5 \cdot 10^{-3}$ & $10^{-12} \ldots 26$ \\
\hline
\end{tabular}

Table 2: Comparison of various ratios of branching ratios in the LHT model, the MSSM and the SM4. From ref. [18].

a strong link between these two observable naturally emerges (see e.g. [10]). In this context, the value $\Delta a_{\mu}=\mathscr{O}\left(10^{-9}\right)$, presently indicated by detailed analyses of $g_{\mu}[11]$, reinforce the expectation of $\mu \rightarrow e \gamma$ within the reach of the MEG experiment.

Beside supersymmetry, there are many other NP models like the Little Higgs model and the Randall-Sundrum models which are able to reach the present bounds and in fact this bounds put already rather stringent constraints on the parameters of these models.

In order to distinguish various NP scenarios, it will be essential to study a large set of decays to three leptons in the final state. Indeed, while in the MSSM $[12,13,14]$ the dominant role in the decays with three leptons in the final state and in $\mu-e$ conversion in nuclei is played by the dipole 
operator, in $[15,16]$ it was found that this operator is much less relevant in the LHT model, with $Z^{0}$ penguin and box diagrams being the dominant contributions. This implies a striking difference between various ratios of branching ratios of type $\operatorname{Br}\left(l_{i} \rightarrow 3 l_{j}\right) / B r\left(l_{i} \rightarrow l_{j} \gamma\right)$ in the MSSM, where they are typically $\mathscr{O}\left(10^{-2}-10^{-3}\right)$ and in the LHT model, where they are $\mathscr{O}\left(10^{-1}\right)$ [17]. The expected correlations among the branching ratios for the most relevant LFV processes are reported in Table 2 from ref. [18].

\section{Conclusions}

The origin of flavour is still, to a large extent, an open question. The synergy and interplay of high energy data with flavour precision data are expected to shed (some) light on this "Flavour Problem". Despite of the remarkable agreement of flavour data with the SM predictions, we still expect New Physics effects to show up in some selected "golden channels" such as LFV processes like $\mu \rightarrow e \gamma$, where an outstanding experimental progress is expected in the upcoming years. Once some clear non-standard effects will be established, the next crucial step will be to trace back the New Physics model at work by means of a careful analysis of correlations among various observables.

\section{References}

[1] N. Cabibbo, Phys. Rev. Lett. 10, 531 (1963); M. Kobayashi and T. Maskawa, Prog. Theor. Phys. 49, 652 (1973).

[2] C. Amsler et al. [ Particle Data Group Collaboration ], Phys. Lett. B667 (2008) 1-1340.

[3] S. Yamada, Search for the lepton flavor violating decay $\mu \rightarrow e \gamma$ in the MEG experiment, Nucl. Phys. Proc. Suppl. 144 (2005) 185-188.

[4] M. Bona et. al., SuperB: A High-Luminosity Asymmetric $e^{+} e^{-}$Super Flavor Factory. Conceptual Design Report, arXiv:0709.0451.

[5] PRISM/PRIME working group Collaboration, Y. Mori et. al., LOI at J-PARC 50-GeV PS, LOI-25, http://psuxl.kek.jp/jhf-np/LOIlist/LOIlist.html.

[6] SINDRUM II. Collaboration, C. Dohmen et. al., Test of lepton flavor conservation in $\mu \rightarrow e$ conversion on titanium, Phys. Lett. B317 (1993) 631-636.

[7] MEGA Collaboration, M. L. Brooks et. al., New limit for the family-number non-conserving decay $\mu^{+} \rightarrow e^{+} \gamma$, Phys. Rev. Lett. 83 (1999) 1521-1524, [hep-ex/9905013].

[8] V. Cirigliano, B. Grinstein, G. Isidori and M. B. Wise, Nucl. Phys. B 728 (2005) 121 [arXiv:hep-ph/0507001].

[9] F. Borzumati and A. Masiero, Phys. Rev. Lett. 57 (1986) 961.

[10] J. Hisano, M. Nagai, P. Paradisi and Y. Shimizu, JHEP 0912 (2009) 030 [arXiv:0904.2080 [hep-ph]].

[11] M. Davier, A. Hoecker, B. Malaescu and Z. Zhang, arXiv:1010.4180 [hep-ph].

[12] J. R. Ellis, J. Hisano, M. Raidal, and Y. Shimizu, Phys. Rev. D66 (2002) 115013.

[13] A. Brignole and A. Rossi, Nucl. Phys. B701 (2004) 3-53.

[14] E. Arganda and M. J. Herrero, Phys. Rev. D73 (2006) 055003. 
[15] M. Blanke, A. J. Buras, B. Duling, A. Poschenrieder, and C. Tarantino, JHEP 05 (2007) 013.

[16] F. del Aguila, J. I. Illana, and M. D. Jenkins, JHEP 01 (2009) 080.

[17] M. Blanke, A. J. Buras, B. Duling, S. Recksiegel and C. Tarantino, Acta Phys. Polon. B 41 (2010) 657 [arXiv:0906.5454 [hep-ph]].

[18] A. J. Buras, B. Duling, T. Feldmann, T. Heidsieck and C. Promberger, JHEP 1009 (2010) 104 [arXiv:1006.5356 [hep-ph]]. 